\title{
5 Implikationen von Mindestmengen und Zertifizierungsvorgaben: Auswirkungen verschiedener Vorgaben auf den deutschen Krankenhaussektor
}

Justus Vogel, Katherine Polin, Christoph Pross und Alexander Geissler

\subsection{Einleitung}

Vorgaben zu Mindestmengen sollen den positiven Zusammenhang zwischen der Anzahl durchgeführter Eingriffe und der erzielten Ergebnisqualität in den stationären Krankenhaussektor übersetzen. Dieser Zusammenhang wurde bereits 1979 erstmalig gezeigt (Luft et al. 1979). Seitdem wurde diese positive Korrelation in zahlreichen Folgestudien bestätigt und gilt mittlerweile für eine Reihe von Leistungsbereichen (LB) als wissenschaftlich gesichert. Der zugrunde liegende Zusammenhang zwischen Menge und Ergebnisqualität wird oftmals in besserer Struktur- und Prozessqualität gesehen (Russo et al. 2010). Weiterhin wird in der Literatur zwischen dem „Practice Makes Perfect (PMP)“und dem „Selective Referral (SR)“-Ansatz unterschieden (Luft et al. 1987; Markar et al. 2012). Der PMP-Ansatz erklärt die bessere Ergebnisqualität durch Lernkurveneffekte, wobei die Anzahl der durchgeführten Eingriffe als Surrogatparameter für die Erfahrung eines Operateurs bzw. eines Krankenhauses angesehen wird. Der SR-Ansatz basiert auf der Annahme, dass Patienten (oder ihre Einweiser) Zentren mit höheren Fallzahlen aufgrund der besseren Ergebnisqualität für ihre Behandlung wählen. In diesen Zentren ist so eine bessere Ausbildung für die Operateure gewährleistet. Darüber hinaus sind diese Zentren aufgrund des Leistungsspektrums attraktiv für qualifizierte Operateure. Mindestmengen sind eine gesundheitspolitische Antwort auf diese wissenschaftlichen Ergebnisse und werden bereits seit geraumer Zeit in der deutschen 
Fachliteratur diskutiert, etwa im Krankenhaus-Report 2002 oder in der Novemberausgabe 2007 der Fachzeitschrift „der Chirurg“. Entsprechend hat der Gemeinsame Bundesausschuss (G-BA) gemäß $\$ 136 f f$. des SGB V bereits 2004 erste LB wie z.B. komplexe Pankreas- und Ösophaguseingriffe in einen Mindestmengenkatalog aufgenommen (Gemeinsamer Bundesausschuss 2018). Der Mindestmengenkatalog wurde in den folgenden Jahren schrittweise erweitert und für einige LB wurde der Schwellenwert der Mindestmengenvorgabe (MMV) angehoben. Schließlich muss gemäß jüngstem Entscheid des Bundesgesundheitsministeriums lediglich noch ein „wahrscheinlicher“ Zusammenhang zwischen der Anzahl durchgeführter Eingriffe und Ergebnisqualität bestehen, um MMV für einen LB festlegen zu können (Hillienhof 2018). Laut aktuellster MMV des G-BA bestehen weiterhin eine Reihe an Übergangs- und Ausnahmeregelungen. Diese Regelungen erlauben es Krankenhäusern, auch bei (prognostizierter) Unterschreitung des definierten Schwellwerts die Leistung weiterhin durchzuführen und abzurechnen, z.B. zur Sicherstellung einer flächendeckenden Versorgung.

Neben den MMV des G-BA bestehen in Deutschland weitere MMV verschiedener Fachgesellschaften zur Zertifizierung von Organ- und Behandlungszentren, die so für hohe Behandlungskompetenz ausgezeichnet werden und den Qualitätswettbewerb verstärken sollen. Darüber hinaus verwenden auch andere Länder in Europa MMV zur stationären Leistungssteuerung, wobei v.a. Unterschiede bei der Art der erlassenden Behörde, der Anzahl der LB, der Anwendungsebene, der Regulierungsabsicht, der Höhe der vorgegebenen Schwellenwerte und den Ausnahmeregelungen bestehen.

Trotz des klaren positiven Zusammenhangs zwischen Fallzahlen und Ergebnisqualität, der zahlreichen Initiativen und des hohen Aufwands zur Mindestmengenregulierung gibt es immer noch zahlreiche Krankenhäuser, die mit relativ geringen Fallzahlen komplexe und spezialisierte Eingriffe durchführen (Peschke et al. 2014). MMV bleiben damit bisher weit hinter ihren vor über 1o Jahren aufgestellten und ambitionierten Zielen zurück und haben ihre volle Wirkung zur Leistungssteuerung noch nicht entfaltet. Vor dem Hintergrund hoher Qualitätsunterschiede, einer mangelnden Spezialisierung und Konzentration sowie Investitionsrückständen und generellem Fachkräftemangel (Sachverständigenrat 2018) müssen die Ausgestaltung, Folgen und strikte Durchsetzung von Mindestmengen stärker in den Fokus der gesundheitspolitischen Diskussion rücken.

Dieser Beitrag soll Informationen und Argumente für diese dringend notwendige Diskussion liefern und analysiert die aktuelle Ausgestaltung und Auswirkungen von MMV entlang der Themenblöcke:

1. Mindestmengenvorgaben in Deutschland

2. Mindestmengenvorgaben in acht ausgewählten europäischen Ländern

3. Auswirkungen einer strikteren Durchsetzung (höherer) Mindestmengenvorgaben auf den deutschen Krankenhaussektor 
Die strukturierte Darstellung der MMV in Deutschland und ausgewählten europäischen Ländern verschafft einen Überblick über den Umfang und den Grad der Anwendung von MMV, wobei auf die MMV in Deutschland detailliert eingegangen wird. Hierbei werden Gemeinsamkeiten und Unterschiede der verschiedenen MMV analysiert und LB für die Modellierung der Auswirkungen einer strikteren Durchsetzung der Mindestmengen identifiziert. Diese Analyse veranschaulicht zweierlei: Erstens besteht in Deutschland weiterhin eine starke Streuung der Leistungserbringung, gerade auch bei komplexen Eingriffen. Zweitens wird ersichtlich, wie viele Krankenhäuser bei einer strikten Durchsetzung verschiedene MMV derzeit erfüllen würden.

\subsection{Methodik}

Die Erstellung dieses Beitrags wurde in folgenden Schritten vorgenommen:

- Es wurde eine Literaturrecherche durchgeführt. Ziele der Literaturrecherche waren das Sammeln und Strukturieren von verschiedenen MMV in Deutschland und die Identifikation von Ländern in Europa, in denen MMV genutzt werden. Außerdem wurden vergleichbare Ansätze zum hier verwendeten Modellierungsansatz untersucht.

- Es wurde eine einfache Modellierung des Effekts einer strikten Durchsetzung von MMV auf die deutsche Krankenhauslandschaft durchgeführt.

\subsubsection{Literaturrecherche}

Zur Identifikation verschiedener MMV in Deutschland wurden die Regelungen des G-BA und verschiedener Fachgesellschaften konsultiert. MMV von anderen europäischen Ländern wurden mittels einer Recherche in wissenschaftlichen Datenbanken (z.B. Pubmed, Google Scholar) und einer Internetrecherche ermittelt. Im Zuge der Recherche wurde ein Mangel an systematischen Reviews zu MMV im internationalen Kontext festgestellt. Angaben zu MMV mussten deshalb teilweise in nationalen Dokumenten in der Landessprache identifiziert und mithilfe von Experteninterviews validiert werden. Fraglos kommen darüber hinaus international weitere MMV zum Einsatz, die jedoch nicht identifiziert werden konnten.

Zur Identifikation der acht Vergleichsländer Dänemark, Finnland, Frankreich, Großbritannien, Italien, Niederlande, Österreich und Schweiz wurde wie folgt vorgegangen: In einem ersten Schritt wurde nach Ländern gesucht, in denen MMV für die gleichen LB wie in Deutschland bestehen. In einem zweiten Schritt wurden Länder, für die nur eine geringe Schnittmenge an LBs identifiziert werden konnte, nicht näher betrachtet und ausgeschlossen (Spanien und Schweden).

Für die Literaturrecherche wurden folgende Begriffe verwendet (auf dänisch, deutsch, englisch, französisch und italienisch): „minimum volume require- 
ments“, „minimum volume regulation“, „minimum volume laws“, „reviews volume outcome relationship“, „minimum volume effects“, „, hospital centralization“, „hospital planning“. Die Suchwörter wurden mit den Namen einzelner Länder verbunden, um landesspezifische Informationen zu erhalten.

\subsubsection{Modellierung}

Für das Untersuchungs- und zuletzt verfügbare Datenjahr 2016 wurde in einem ersten Schritt folgendes analysiert:

- Anzahl der Krankenhäuser, die mind. 1 Eingriff des jeweiligen LBs durchführten, und die durchschnittliche Anzahl durchgeführter Eingriffe je Krankenhaus

- Anzahl der Krankenhäuser, die die jeweilige MMV erbringen, und die durchschnittliche Anzahl der durchgeführten Eingriffe in diesen Krankenhäusern

Als Dateninput wurden die von den Krankenhäusern in den strukturierten Qualitätsberichten der externen stationären Qualitätssicherung für das Untersuchungsjahr 2016 angegebenen OPS-Fallzahlen verwendet. Hierbei wurden Krankenhäuser über die Kombination von Institutionskennzeichen und Standortnummer identifiziert, d.h. jeder Krankenhausstandort wurde als ein „Krankenhaus“ gezählt (vgl. gesetzliche Vorgabe zum Gültigkeitsbereich von Mindestmengen $\mathbb{1 3 6 b}$ Abs. 1 Satz 1 Nr. 2 SGB V und G-BA 2018). Zur Kategorisierung der OPS-Codes in LB wurden die Abgrenzungen bzw. Definitionen des G-BA, der Deutschen Gesellschaft für Allgemein- und Viszeralchirurgie (DGAV), der Deutschen Krebsgesellschaft (DKC), des Wissenschaftlichen Instituts der AOK (WIdO) und des Disease Management Programms (DMP) für Brustkrebs herangezogen. Die Definitionen unterscheiden sich hinsichtlich ihres Umfangs (Anzahl der OPS-Codes und Eingriffsarten je LB) und hinsichtlich ihrer Detailtiefe (Angabe als 4-, 5- oder 6-Steller). Für eine Übersicht der verwendeten Definitionen je LB s. Tab. 1.

In den strukturierten Qualitätsberichten liegen lediglich die Summen der OPSbzw. ICD-Codes auf Fachabteilungs- bzw. Krankenhausebene vor. Daten auf Patientenebene, d.h. ICD-Code (Hauptdiagnose) in Kombination mit erfolgter Behandlung als OPS-Code(s) auf Patientenebene, sind nicht verfügbar. Deshalb werden in der Modellierung ausschließlich OPS-Codes zur Definition der LB verwendet. Die dadurch entstehende Datenungenauigkeit und das durch den Datenschutz bedingte Fehlen von Fallzahlen $\leq 3$ Eingriffe wurden für die durchgeführte Analyse als akzeptabel angesehen (OPS-Fallzahlen $\leq 3$ werden als 1 Eingriff gezählt).

Für LB, für die mehr als eine Definition besteht, wurde die obige Modellierung für jede Definition durchgeführt. Die Ergebnisse dieses ersten Modellierungsschritts werden im folgenden Kapitel vorgestellt. 
5 Implikationen von Mindestmengen und Zertifizierungsvorgaben:

Auswirkungen verschiedener Vorgaben auf den deutschen Krankenhaussektor

Tab. 1 Definition und Abgrenzung der Leistungsbereiche anhand von ICD- und OPS-Codes gemäß G-BA, DGAV, DKG, WIdO und DMP

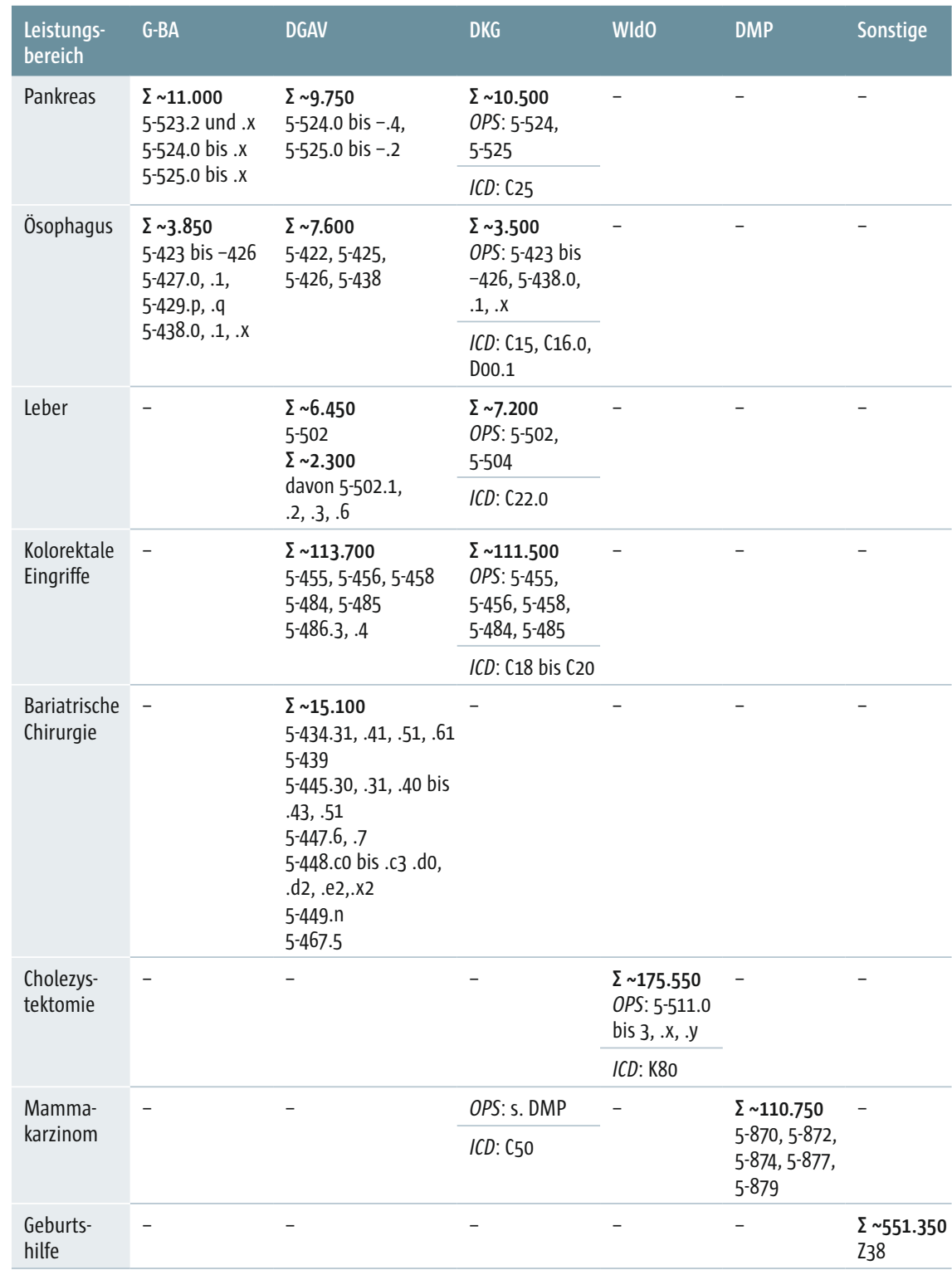

Hinweise: Nur zur Modellierung verwendete LB angegeben. ICD-Codes wurden mit aufgenommen, um die vollständigen Definitionen darzustellen, ICD-Codes wurden jedoch nicht zur Modellierung verwendet (Ausnahme: Geburtshilfe). Die Definition für den LB Geburtshilfe wurde eigenständig vorgenommen. Es wird immer die höchste Aggregationsebene verwendeter Codes angegeben (z.B. 5-511.0 anstatt 5-511.01 und 5-511.02 oder 5-423 statt 5-423.0 bis .y). Die Gesamtzahl der in 2016 von den in der Stichprobe enthaltenen Krankenhäusern durchgeführten Eingriffe ist nach dem Summenzeichen auf volle 50-er gerundet angegeben.

Quellen für Definition der LB: Blum und Offermanns (2006), Gemeinsamer Bundesausschuss (2018), DGAV e.V. (2018), Onkozert (2018), WIdO (2017)

Datenquelle: Strukturierte Qualitätsberichte der externen stationären Qualitätssicherung 2016

urheberrechtlich geschützt, 
In einem zweiten Schritt wurde für den deutschen Krankenhaussektor dargestellt, wie viele Krankenhäuser die MMV anderer europäischer Länder erfüllen. Die Ergebnisse dieses Modellierungsschritts werden im vierten Abschnitt dargestellt.

Loos et al. (2016) haben eine ähnliche Modellierung für den deutschen Krankenhaussektor unter Berücksichtigung der Auswirkungen auf die durchschnittliche Erreichbarkeit vorgenommen. Schwellenwerte leiten die Autoren aus der Fachliteratur ab. Im Unterschied zu Loos et al. werden im vorliegenden Beitrag die Schwellenwerte zur Modellierung aus dem Vergleich von in Deutschland und anderen europäischen Ländern gültigen MMV abgeleitet und Implikationen auf die Erreichbarkeit oder die Patientenfahrzeit werden nicht näher beleuchtet. Weitere mögliche Weiterentwicklungen der hier durchgeführten einfachen Modellierung sind (1.) Berechnung der Auswirkungen auf die Ergebnisqualität (z.B. in Form von Mortalität), (2.) Umverteilung der Patienten von Krankenhäusern, die von der Versorgung ausgeschlossen werden und (3.) Berücksichtigung von Kapazitätsbedingungen und-verschiebungen. Arbeiten, die sich selektiv mit den obigen Weiterentwicklungen befassen, sind beispielsweise Loos et al. (2016) und Geraedts et al. (2010) für Erreichbarkeit bzw. Patientenreisezeit sowie Nimptsch u. Mansky (2018) für Ergebnisqualität und Patientenreisezeit. Außerdem wurden von de Cruppé et al. (2014) und de Cruppé und Geraedts (2016 und 2018) umfassende Studien zur Einhaltung von G-BA-MMV für die Datenjahre 2004 bis 2010, 2006 bis 2010 bzw. 2006 bis 2014 durchgeführt. Darüber hinaus wurde eine systematische Übersichtsarbeit zum Zusammenhang zwischen Menge und Qualität unter anderem von Chowdhury et al. (2007) vorgelegt.

\subsection{Mindestmengen- und Zertifizierungsvorgaben in Deutschland}

Unterschiedliche Organisationen geben in Deutschland unterschiedlich hohe und restriktive MMV für unterschiedliche Zwecke vor. MMV, die direkte Auswirkungen auf die Vergütung von Krankenhäusern haben und „Gelegenheitschirurgie“ eindämmen sollen, werden durch den G-BA bestimmt. Diese MMV sind ein direkter, regulativer Eingriff in die Versorgungsstruktur durch einen mittelfristigen Ausschluss von Krankenhäusern von der Versorgung. Fachgesellschaften definieren darüber hinaus MMV zur (Re-)Zertifizierung von Organoder Behandlungszentren (z.B. Darmkrebszentrum der DKG oder Zentrum für Endoskopie der DGAV). Zertifizierungsvorgaben sollen Spitzenmedizin anzeigen sowie Zentren mit hoher Qualität auszeichnen und so den Qualitätswettbewerb nachhaltig verstärken. Ziel ist eine langfristige Verlagerung der Patientenströme hin zu zertifizierten Zentren, wobei Krankenhäuser mit niedrigen Fallzahlen nicht zwangsweise von der Versorgung ausgeschlossen werden. Während die MMV des G-BA für alle Krankenhäuser rechtlich bindend sind, müssen nur solche Krankenhäuser, die eine (Re-)Zertifizierung einer bestimmten Fachgesellschaft erlangen wollen, entsprechende MMV berücksichtigen.

urheberrechtlich geschützt, 


\subsubsection{Mindestmengenvorgaben des G-BA}

Der aktuellste durch den G-BA erarbeitete Mindestmengenkatalog umfasst acht LB (G-BA 2018). Die Anzahl der Eingriffe bzw. Fälle pro LB sind jährlich pro Standort eines Krankenhauses zu erbringen und nachzuweisen:

1. Lebertransplantation: 20

2. Nierentransplantation: 25

3. Komplexe Eingriffe am Organsystem Ösophagus: 10

4. Komplexe Eingriffe am Organsystem Pankreas: 10

5. Stammzellentransplantation: 25

6. Kniegelenk-Totalendoprothesen: 50

7. Koronarchirurgische Eingriffe: ohne die Festlegung einer konkreten Mindestmenge

8. Versorgung von Früh- und Neugeborenen mit einem Geburtsgewicht von < $1.250 \mathrm{~g}: 14$

Die Zählung und der entsprechende Nachweis der erbrachten Fallzahl je LB basiert auf einer vom G-BA festgelegten Auswahl an OPS-Codes, wobei für koronarchirurgische Eingriffe bisher keine Mindestmenge und keine OPS-Codes festgelegt wurden. Damit ein Krankenhaus Behandlungen in den obigen LB durchführen darf, muss das Krankenhaus eine berechtigte Prognose der erwarteten Behandlungsmenge basierend auf dem letzten Kalenderjahr bei den Landesverbänden der Krankenkassen und Ersatzkassen einreichen. Eine positive Prognose ohne Erreichen der Mindestmenge im vorangegangen Kalenderjahr kann hier mit personellen, strukturellen und/oder sonstigen Veränderungen begründet werden. Sollte keine positive Prognose abgegeben werden können bzw. eine positive Prognose nicht akzeptiert werden, wird das entsprechende Krankenhaus von der Versorgung ausgeschlossen und erhält im Falle einer dennoch durchgeführten Behandlung keine Vergütung. Eine positive Prognose ohne Erreichen der Mindestmenge im vorangegangen Leistungsjahr kann darüber hinaus dann beschieden werden, wenn das Krankenhaus einen Ausnahmetatbestand oder eine Übergangsregelung geltend machen kann. Als Ausnahmetatbestand kann entweder vorgebracht werden, dass die Leistung erstmalig bzw. erneut (mind. 24 Monate Unterbrechung) erbracht wird oder, dass die erbrachte Qualität den Anforderungen und Kriterien des G-BA für die entsprechende Leistung entspricht. Darüber hinaus greifen die MMV bei Neueinführung oder Erhöhung einer Mindestmenge auf Krankenhausebene oder Einführung eines Arztbezugs für einen LB bis zu 24 Monate i.S.e. Übergangsregelung nicht und die Leistung kann ohne Einschränkungen erbracht werden.

\subsubsection{Mindestmengenvorgaben durch Fachgesellschaften}

Neben den MMV des G-BA bestehen MMV für die Zertifizierung von Organund Behandlungszentren verschiedener Fachgesellschaften. In diesem Beitrag 
wird beispielhaft auf die MMV der DGAV und der DKG eingegangen, da beide Fachgesellschaften v.a. chirurgische LB fokussieren, für die ein ausgeprägter Mengen-Qualitäts-Zusammenhang besteht und die deshalb für die Modellierung verwendet werden.

Insgesamt bietet die DGAV Zertifizierungen für elf verschiedene Zentren (DGAV e.V. 2018), wobei für jedes Zentrum zwischen den Leveln Kompetenz-, Referenz- und Exzellenzzentrum unterschieden wird. Für Kompetenzzentren gelten die niedrigsten und für Exzellenzzentren die höchsten Anforderungen, welche von der DGAV und ggf. kooperierenden Fachgesellschaften (z.B. der Deutschen Hernien Gesellschaft) erarbeitet werden. Eine für alle Zertifizierungen der DGAV gültige Anforderung ist der Nachweis von Mindestmengen für den LB des Zentrums. Dieser LB wird anhand einer festgelegten Auswahl an OPS-Codes definiert. Hierbei ist zu beachten, dass die DGAV mehrere Eingriffsarten innerhalb eines LBs definiert. Je nach LB bestehen Schwellenwerte entweder separat für jede Eingriffsart oder für die Summe der Eingriffe aller Eingriffsarten. Beispielsweise werden vier Eingriffsarten für das Zentrum für Chirurgische Erkrankungen des Pankreas mit jeweils einem Schwellenwert je Eingriffsart definiert (z.B. 25 Pankreaskopfresektionen und [totale] Pankreatektomien für Kompetenzzentren). Ein Beispiel für den Nachweis der Summe der Eingriffe aller Eingriffsarten ist die (Re-)Zertifizierung als Zentrum für Adipositas- und metabolische Chirurgie: Hierfür muss ein Kompetenzzentrum insgesamt die Summe von 50 Eingriffen pro Jahr in acht Eingriffsarten (u.a. Magenband) nachweisen.

Der Nachweis der geforderten Mindestmenge muss bei Erstzertifizierung für die letzten zwei Kalenderjahre und bei Rezertifizierung für die letzten drei Kalenderjahre vor Antragsstellung erfolgen. Der Nachweis wird anhand von Vorlage der durchgeführten Eingriffe je Eingriffsart erbracht. Generell bestehen keine Ausnahmen für ein Nichterreichen der geforderten Mindestmengen in der definierten Anzahl an Kalenderjahren vor (Re-)Zertifizierung. Jedoch kann bei einer Unterschreitung der Mindestmenge in einer Eingriffsart in einem Kalenderjahr auch der Durchschnitt der letzten zwei Kalenderjahre bei Erstzertifizierung bzw. der letzten fünf Kalenderjahre bei Rezertifizierung zur Einhaltung der Mindestmenge in dieser Eingriffsart herangezogen werden.

Neben der DGAV ist auch die DKG in der Zertifizierung von Zentren aktiv. Insgesamt können acht Zertifizierungen für verschiedene Organ(-systeme), wie zum Beispiel Darm oder Lunge, erworben werden (Onkozert 2018). Darüber hinaus werden für das onkologische und das viszeralonkologische Zentrum mehrere Zusatzmodule angeboten (z.B. Kinderonkologie oder Leber). Die Zertifizierungsvorgaben für jedes Zentrum werden von Fachgruppen innerhalb einer interdisziplinären Zertifizierungskommission erarbeitet. Für den Croßteil der LB werden MMV auf Ebene von ICD- und/oder OPS-Codes definiert. Beispielsweise muss ein viszeralonkologisches Zentrum für die (Re-)Zertifizierung des Moduls Leber eine jährliche Mindestmenge von 30 Leberkarzinomen (Primärdiagnose) anhand des ICD-Codes C22.0 nachweisen. Darüber hinaus 
müssen jährlich mindestens 25 Leberresektionen und/oder -transplantationen durchgeführt werden (OPS-Codes 5-502 und 5-504). Grund für den Nachweis sowohl auf ICD- wie auch auf OPS-Code-Ebene ist, dass die DKC Zentren mit nachgewiesener Kompetenz in der Krebsbehandlung auszeichnen möchte. Hierfür ist eine Identifizierung der der Behandlung zugrunde liegenden Hauptdiagnose unerlässlich. Die DGAV beschränkt sich lediglich auf den Nachweis anhand von OPS-Codes, da die Kompetenz eines Zentrums für eine bestimmte Behandlung bzw. einen Eingriffsbereich (z.B. Endoskopie oder Hernienchirurgie) oder ein Organ(-system) (z.B. Pankreas oder Speiseröhre und Magen) unabhängig von der Diagnose ausgewiesen werden soll.

\subsubsection{Vergleich deutscher Mindestmengenvorgaben}

Für einige LB bestehen MMV von mehreren Organisationen. Hierbei gilt es zu beachten, dass (1.) der beabsichtigte Zweck der MMV variiert, (2.) entsprechend der (rechtliche) Geltungsbereich der MMV unterschiedlich ist (s.o. in der Einleitung zu 5.3.), (3.) MMV auf verschiedenen Ebenen (Krankenhaus und/oder Operateur) vorgegeben werden und außerdem (4.) die Höhe der Schwellenwerte anders gesetzt ist. Weiterhin deuten (5.) zwar die Klarnamen der LB auf Gemeinsamkeiten hin, die Abgrenzung anhand von ICD- und/oder OPS-Codes ist jedoch nicht deckungsgleich. Beispielsweise umfassen für den LB Pankreas sowohl die MMV des G-BA wie auch der DGAV OPS-Codes für verschiedene partielle Resektionen des Pankreas und (totale) Pankreatektomien, jedoch inkludiert der G-BA zusätzlich zwei 5-stellige OPS-Codes zur inneren Drainage des Pankreas in seiner MMV (s. Tab. 1). Die DGAV inkludiert die innere Drainage des Pankreas auch in ihren MMV, jedoch nicht in der gleichen Eingriffsart wie partielle Resektionen des Pankreas bzw. wie (totale) Pankreatektomien, sondern in der Eingriffsart Weitere Eingriffe am Pankreas. Darüber hinaus inkludiert die DGAV alle inneren Drainagen, d.h. OPS-Code 5-523, in ihrer MMV. Außerdem gehören der Eingriffsart Weitere Eingriffe am Pankreas noch sechs weitere 5-stellige und mehr als ein Dutzend 6-stellige OPS-Codes an. Schließlich inkludiert die DGAV für den LB Pankreas noch die Eingriffsart interventionelle Therapien (z.B. Abszessdrainage) in ihrer MMV. Weitere Unterschiede in den Definitionen der LB sind der Detailgrad einzelner OPS-Codes (4-, $5^{-}$ und 6-stellige OPS-Codes) und verschiedene Definitionen gleicher Eingriffsarten (z.B. [totale] Pankreatektomie umfasst drei OPS-Codes mehr in der G-BA$M M V$ als in der DGAV-MMV). Außerdem macht die DGAV für einige Zentren MMV für zusammenhängende Organsysteme (z.B. Ösophagus und Magen) bzw. definiert auch für diagnostische Leistungen MMV (z.B. für Koloproktologie). Demgegenüber definiert die DKG LB in den meisten Fällen neben OPS-Codes für den Nachweis der operativen Expertise zusätzlich über eine Kombination von ICD- und OPS-Codes oder nur ICD-Codes zum Nachweis von Primärfällen. So umfassen die DKG-MMV für den LB Pankreas eine operative Expertise von mind. 20 Pankreasresektionen sowie 12 operativen Primärfällen (für genaue 
Definition s. Tab. 1). Die Eingriffe der operativen Expertise sind für den LB Pankreas an keine Diagnose gekoppelt und können auch für benigne Tumore durchgeführt worden sein. Für den Großteil der MMV werden 4-stellige OPSCodes und 3-stellige ICD-Codes verwendet. Im Übrigen fordert die DKG Mindestmengen sowohl auf Krankenhaus- wie auch zusätzlich auf Operateurebene, während G-BA und DGAV nur MMV auf Krankenhausebene festlegen.

Abschließend bleibt festzustellen, dass die den MMV zugrunde liegende Logik jedoch in allen Fällen dieselbe ist und auf der in der Einleitung beschriebenen Korrelation zwischen Ergebnisqualität und der Anzahl der durchgeführten Eingriffe bzw. Behandlungen fußt.

\subsubsection{Modellierung deutscher Mindestmengenvorgaben}

Der im Abschnitt Methodik skizzierte erste Modellierungsschritt wird entlang drei verschiedener MMV (G-BA, DGAV, DKG) und entlang vier LB (Pankreas, Ösophagus, Leber, Kolorektale Eingriffe) dargestellt. Zur Modellierung wurden diejenigen LB ausgewählt, für die aufgrund verschiedener Definitionen und/oder MMV unterschiedliche Auswirkungen auf den deutschen Krankenhaussektor gegeben wären. Von den DGAV-MMV wurde(n) die Eingriffsart(en) ausgewählt, die mit den MMV des G-BA und/oder der DKG am ehesten vergleichbar sind. Außerdem werden die DGAV-Schwellenwerte für Kompetenzzentren angewendet.

Im Untersuchungsjahr führten 618 bzw. 610 Krankenhäuser Eingriffe der Definition für den LB Pankreas des G-BA bzw. der DKG durch. Demgegenüber fallen nur 460 Krankenhäuser unter die Definition der DGAV, was daran liegt, dass die DGAV einerseits den LB enger definiert und andererseits nur Krankenhäuser gezählt wurden, die jeweils mind. einen Eingriff der zwei fokussierten Eingriffsarten erbracht hatten (s. Abb. 1). Lediglich 66 Krankenhäuser entsprechen der DGAV MMV, was am relativ hohen Schwellenwert und MMV für zwei separate Eingriffsarten liegt (25 Pankreaskopfresektion und totale Pankreatektomien, 5 Pankreaslinks- und Segmentresektionen). Demgegenüber entspricht mehr als die Hälfte der Krankenhäuser der G-BA-MMV.

Auch für den LB Ösophagus ist die Anzahl der Krankenhäuser nach G-BA-Definition mit der Anzahl der Krankenhäuser nach DKG-Definition vergleichbar. Die Anzahl der Krankenhäuser nach DGAV-Definition ist demgegenüber um mehr als 100 Krankenhäuser geringer. Gründe hierfür sind, dass nur Krankenhäuser gezählt wurden, die jeweils mindestens einen Eingriff beider fokussierten Eingriffsarten durchführten (vgl. Pankreas), und dass der LB wesentlich anders definiert ist (s. Tab. 1). Auch für Ösophagus fordert die DGAV MMV für zwei Eingriffsarten (15 Ösophagusresektionen bei Malignom und 5 sonstige Eingriffe am Ösophagus). In Summe inkludiert die DGAV ca. doppelt so viele Eingriffe wie die DKG und der G-BA (s. Tab. 1), die kombiniert mit der geringeren Anzahl an Krankenhäusern in einer ca . 2,6-mal höheren durchschnittlichen Anzahl an Eingriffen je Krankenhaus resultiert (ohne MMV). Es erfül-

urheberrechtlich geschützt, 
5 Implikationen von Mindestmengen und Zertifizierungsvorgaben:
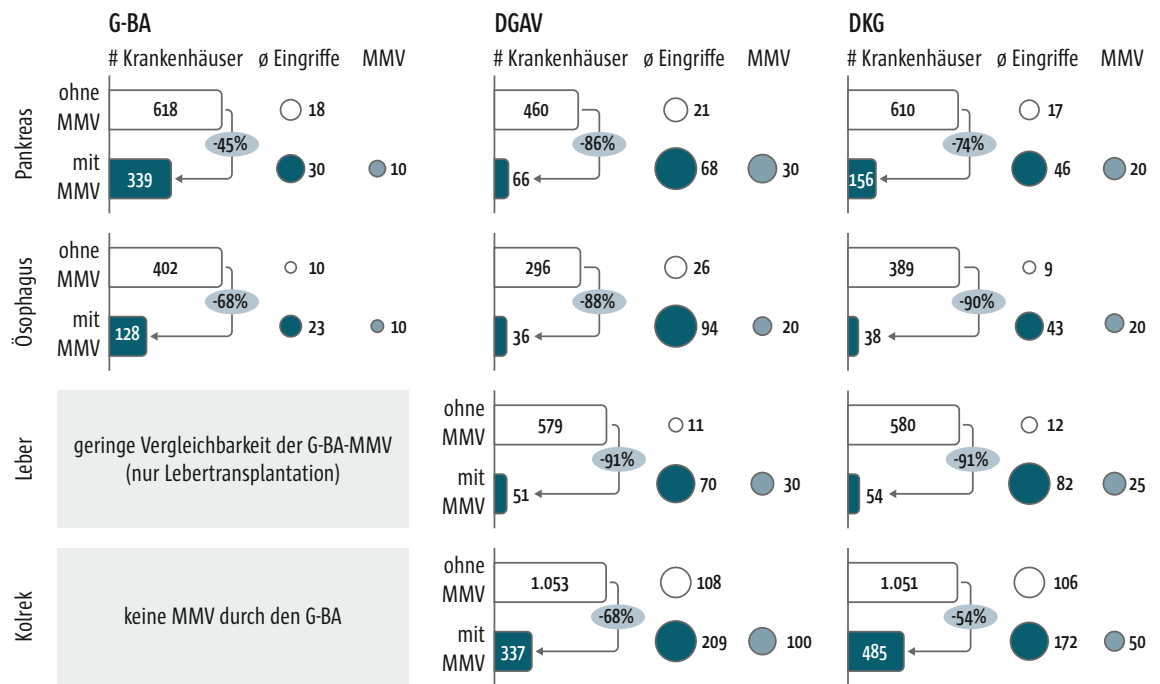

Abb. 1 Anzahl an Krankenhäusern, die unterschiedlichen deutschen MMV auf Krankenhausebene entsprechen. Hinweise: (1.) Die durchschnittliche Anzahl der Eingriffe für „mit MMV“ bezieht sich nur auf die Eingriffe, die von den Krankenhäusern tatsächlich erbracht wurden. Eingriffe, die von Krankenhäusern erbracht wurden, die der MMV nicht entsprechen, wurden nicht auf die verbleibenden Krankenhäuser verteilt. (2.) Für die MMV der DKG handelt es sich lediglich um eine Abbildung nach Teilkriterien (OPS-Codes). Für eine vollständige Darstellung sind ICD- und OPS-Codes auf Patientenebene nötig.

len ca. 3,5-mal so viele Krankenhäuser die G-BA-MMV wie die DGAV-bzw. die DKG-MMV. Jedoch ist der Anteil der Krankenhäuser, der die verschiedenen MMV für Ösophagus erfüllt, generell kleiner als für Pankreas und mit jeweils um die 10\% im Falle der DGAV- und der DKG-MMV insgesamt sehr gering. Entsprechend ist die durchschnittliche Anzahl durchgeführter Eingriffe je Krankenhaus jeweils ca. 4-mal so hoch wie ohne MMV, während die G-BA-MMV geringere Auswirkungen auf die durchschnittliche Anzahl der Eingriffe hat (ca. 2,4-mal so hoch wie ohne MMV). Trotzdem wird deutlich, dass sowohl für Pankreas wie auch für Ösophagus eine strikte Anwendung der G-BA-MMV starke Zentralisierungseffekte hätte und „Gelegenheitschirurgie“ unterbunden würde. Außerdem erfüllt ein signifikanter Anteil an Krankenhäusern die G-BAMMV nicht, was zumindest teilweise durch die Ausnahmeregelungen zu erklären ist (vgl. de Cruppé u. Geraedts 2018).

Für den LB Leber wurden nur solche MMV verglichen, die anatomische (typische) Leberresektionen miteinschließen. Da die G-BA-MMV lediglich Teilresektionen und Hepatektomien zur Transplantation und Transplantationen an sich umfasst, wird die G-BA-MMV hier nicht mit betrachtet. Die DGAV inkludiert nur anatomische Leberresektionen in ihrer MMV während die DKG zusätzlich Lebertransplantationen akzeptiert (s. Tab. 1). Entsprechend ist die durchschnittliche Anzahl an Eingriffen trotz beinahe gleicher Anzahl der

urheberrechtlich geschützt, 
Krankenhäuser für die DKG-MMV höher als für die DGAV-MMV (s. Tab. 1). Obwohl die DGAV für anatomische Leberresektionen allgemein eine MMV von 25 Eingriffen und zusätzlich davon mindestens 5 Resektionen von 4 und mehr Segmenten der Leber fordert, entsprechen nur unwesentlich weniger Krankenhäuser dieser MMV als der DKG-MMV. Insgesamt entsprechen weniger als 10\% der Krankenhäuser der DGAV- bzw. der DKG-MMV.

Da die DGAV- und die DKG-Definitionen für den LB Kolorektale Eingriffe beinahe deckungsgleich sind, fällt quasi die gleiche Anzahl an Krankenhäusern unter beide Definitionen. Obwohl der DGAV-Schwellenwert doppelt so hoch ist wie der DKG-Schwellenwert, ist die Anzahl der Krankenhäuser, die die DKGMMV einhalten, nur ca. 44\% höher. Entsprechend ist die Anzahl der durchschnittlich durchgeführten Eingriffe je Krankenhaus für die DGAV-MMV lediglich um ca. 22\% höher. Hieraus kann gefolgert werden, dass der Grad der Zentralisierung weniger stark zunimmt als der Schwellenwert. Dies deutet darauf hin, dass auch ohne MMV bereits viele Krankenhäuser eine relativ hohe Menge an Eingriffen durchführen. Dass mit ca. 32\% bzw. 46\% relativ viele Krankenhäuser der DGAV- bzw. der DKG-MMV entsprechen, unterstützt die obige Schlussfolgerung zusätzlich. Im Übrigen definiert sowohl die DGAV als auch die DKG separate Schwellenwerte für die Eingriffsarten Kolonresektion (75 bzw. 30 Eingriffe) und Rektumresektion (25 bzw. 20 Eingriffe).

Abschließend kann festgestellt werden, dass weder die G-BA-, oder die DGAV-, noch die DKG-MMV von einem signifikanten Anteil der Krankenhäuser in Deutschland eingehalten werden. Hierbei ist der Anteil, der die G-BA-MMV einhält, am größten (ca. 55\% für Pankreas und ca. $32 \%$ für Ösophagus), während die DGAV-MMV generell vom geringsten Anteil der Krankenhäuser eingehalten werden. Dies entspricht den Absichten der verschiedenen MMV: Während der G-BA an einer Vermeidung von Gelegenheitschirurgie interessiert ist, über die Vergütung direkt in Versorgungsstrukturen eingreift und darüber hinaus eine flächendeckende Versorgung berücksichtigen muss, möchten DGAV und DKG (wenige) Zentren der Spitzenmedizin auszeichnen, so Qualitätstransparenz und -wettbewerb stärken und eine natürliche, langfristige Steuerung der Patientenströme erreichen.

Loos et al. (2016) betrachten in ihrer Modellierung andere LB als die hier analysierten LB, weshalb ein direkter Vergleich der Ergebnisse nicht gegeben ist. Außerdem verwenden die Autoren zwar ebenfalls die strukturierten Qualitätsberichte der externen Qualitätssicherung als Datenbasis, untersuchen jedoch das Jahr 2014. Insgesamt lässt sich aber feststellen, dass der Grad der Leistungserbringer, die eine $M M V$ einhalten bzw. nicht einhalten, vergleichbar mit den oben vorgestellten Ergebnissen ist (z.B. 38\% der Krankenhäuser erfüllen eine MMV von 40 radikalen Prostatovesikulektomien). Die Forschergruppe um de Cruppé und Geraedts untersuchen teilweise die gleichen LB wie die hier analysierten LB und nutzen ebenfalls die strukturierten Qualitätsberichte der externen stationären Qualitätssicherung als Datenbasis, jedoch für andere Datenjahre (s. Kap. 5.2). Wie oben gezeigt, finden die Autoren ebenfalls, dass 
ein größerer Anteil an Krankenhäusern die G-BA-MMV für Pankreas einhält als für Ösophagus (de Cruppé u. Geraedts 2018). Der Anteil der Krankenhäuser, die die G-BA-MMV einhält, ist für die von den Autoren untersuchten Datenjahre vergleichbar zu dem hier für 2016 gefundenen, der Einhaltungsgrad ist insgesamt jedoch höher (zwischen 50 und 60\% für Ösophagus und zwischen 65 und $75 \%$ für Pankreas). Erklärung hierfür könnte sein, dass generell Unterschiede zwischen den verschiedenen Informationskategorien der Qualitätsberichte bestehen (z.B. OPS-Fallzahlen gegenüber [Selbst-]Angabe der Krankenhäuser zu Einhaltung von Mindestmengen). Bei Verwendung der gleichen Informationskategorie können Unterschiede zumindest teilweise durch verschiedene Zählweisen für nicht gemeldete Fälle $\leq 3$ und/oder die Zählweise einzelner Krankenhausstandorte erklärt werden (s. Kap. 5.2). Im Übrigen ist die von de Cruppé und Geraedts untersuchte Stichprobe nicht direkt mit der hier analysierten Stichprobe an Krankenhäusern vergleichbar, da die Autoren die durch die Krankenhäuser vorgebrachten Begründungen der Nicht-Einhaltung einer MMV untersuchen und nicht die Einhaltungsgrade verschiedener MMV an sich.

Demgegenüber haben Mansky et al. (2016) für das Datenjahr 2015 Einhaltungsraten von ca. $36 \%$ für Ösophagus und 55\% für Pankreas im Rahmen einer Auswertung der German Inpatient Quality Indicators errechnet, welche für Ösophagus nur gering bzw. für Pankreas fast gar nicht von den oben dargestellten Einhaltungsraten für 2016 abweichen.

\subsection{Mindestmengenvorgaben in Europa}

Im Folgenden wird eine Übersicht über die MMV in acht europäischen Ländern angeführt: Dänemark, Finnland, Frankreich, Großbritannien, Italien, Niederlande, Österreich und Schweiz. Für jedes Land werden die MMV für insgesamt acht LB der Allgemein-/Viszeralchirurgie und der Mutter-Kind-Versorgung tabellarisch vorgestellt (Pankreas, Ösophagus, Leber, Kolorektale Eingriffe, Bariatrische Eingriffe, Cholezystektomie, Mammakarzinom, Geburtshilfe). Diese LB wurden ausgewählt, da hier eine große Überschneidung zwischen den untersuchten Ländern und Deutschland gefunden werden konnte (Ausnahme: Cholezystektomie). Relevante Unterschiede im Vergleich zu MMV in Deutschland werden diskutiert. Abschließend wird die Anwendung der MMV der europäischen Vergleichsländer für die deutsche Versorgungslandschaft für alle LB dargestellt.

\subsection{1 Übersicht und Vergleich}

Für eine Übersicht der MMV je LB und Land s. Tab. 2. Die Rechercheergebnisse je Land werden entlang folgender sechs Dimension verglichen: (1.) Art der erlassenden Behörde, (2.) Anzahl der LB mit MMV, (3.) Anwendungsebene der MMV (Krankenhaus vs. Operateur), (4.) Regulierungsabsicht, (5.) Höhe der 


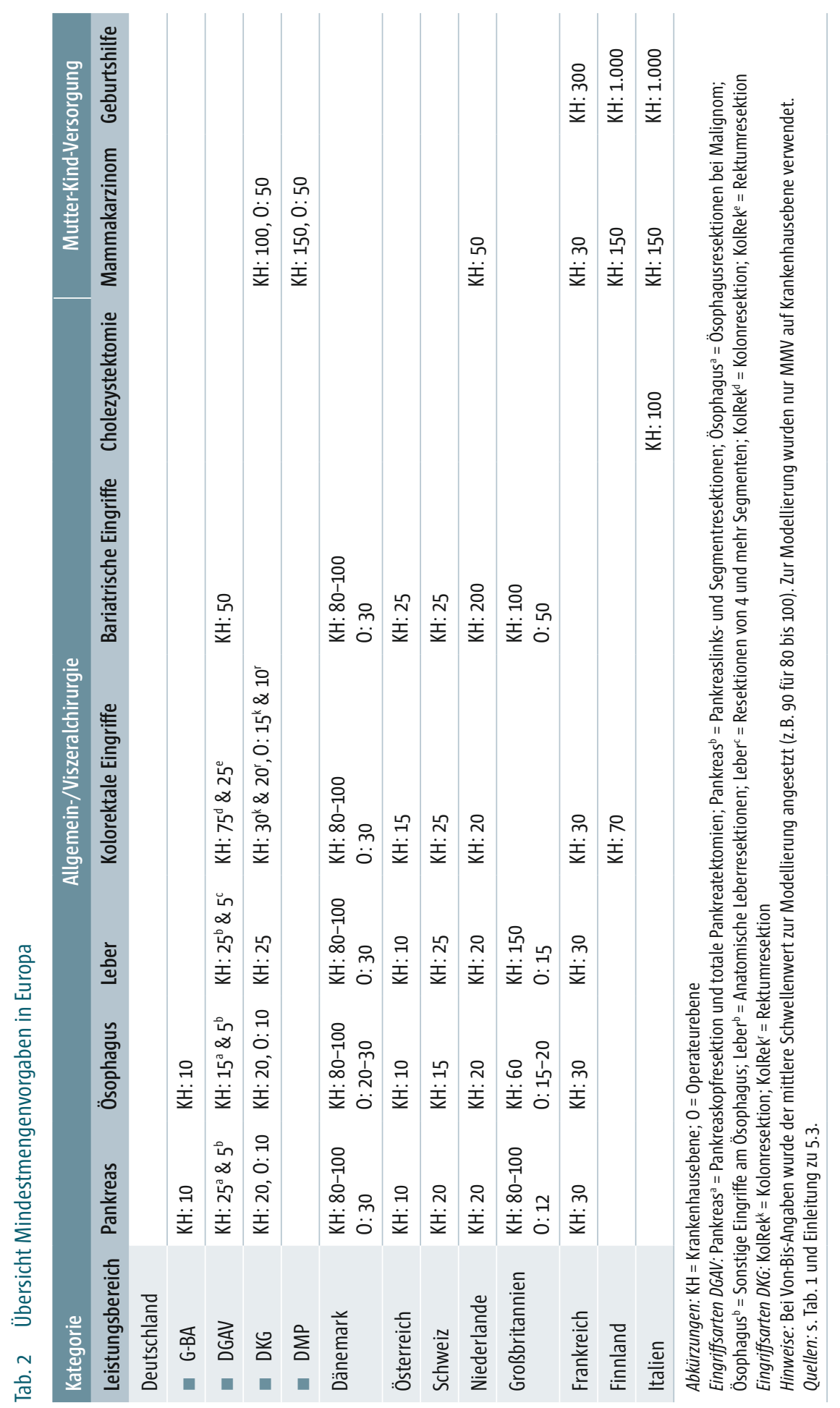

urheberrechtlich geschützt, 
Schwellenwerte, (6.) Grad der Durchsetzung und Ausnahmeregelungen (Allum et al. 2011; AUGIS 2010; Geissler et al. 2017; Italia 2012, 2015; Jüni et al. 2014; KCE 2017; Nederlandse 2017; Nederlandse Vereniging voor Heelkunde 2017; NHS 2018; Nobilio et al. 2004; ÖSG 2017; République Française 2011; SPLG 2018; Suomi 2017; Zorguerzekeraars Nederland 2017).

\section{Art der erlassenden Behörde}

In den acht Vergleichsländern werden MMV sowohl von nationalen wie auch regionalen Behörden festgelegt. Darüber hinaus definieren in den untersuchten Ländern ähnlich wie in Deutschland Fachgesellschaften MMV und in manchen Fällen werden MMV direkt von Krankenversicherungen festgelegt. In Österreich und Frankreich werden MMV ausschließlich von den Bundesministerien für Gesundheit erlassen. Demgegenüber werden in Deutschland wie bereits gesehen MMV einerseits mit bundesweiter Gültigkeit durch den G-BA und auf freiwilliger Basis durch die med. Fachgesellschaften definiert. In der Schweiz werden MMV für komplexe LBs (vgl. Tab. 1) durch die Interkantonale Vereinbarung über die hochspezialisierte Medizin erlassen. Zusätzlich sind jedoch die kantonalen Direktorate für die Krankenhauskapazitätsplanung und die Erstellung der sogenannten Spitalliste verantwortlich, wofür zusätzliche MMV festgelegt und verwendet werden können. Entsprechend variiert die Höhe der Schwellenwerte gleicher LBs entlang der Bevölkerungsdichte und den damit verbundenen Anforderungen an die Versorgungsdichte je Kanton. In Großbritannien und den Niederlanden werden MMV von den Fachgesellschaften entwickelt. Jedoch legen in den Niederlanden auch Krankenversicherungen MMV fest, deren Schwellenwerte generell höher liegen als die der Fachgesellschaften. Jedoch gelten diese MMV nur für Krankenhäuser, die bei der jeweiligen Krankenversicherung versicherte Patienten behandeln.

\section{Anzahl der Leistungsbereiche mit MMV}

Kein Land hat für alle acht fokussierten LB MMV definiert. In Frankreich und den Niederlanden bestehen mit sechs LB die meisten MMV. In Deutschland bestehen inkl. Zertifizierungsvorgaben ebenfalls MMV für sechs LB. In Finnland und Italien bestehen MMV für drei LB und damit insgesamt am wenigsten MMV für die Fokuskategorien Allgemein-/Viszeralchirurgie und Mutter-Kind-Versorgung. Jedoch gilt zu beachten, dass Finnland und Italien für andere LB weitere MMV definiert haben (z.B. Gelenkersatz Schulter in Finnland oder Herzinfarkt in Italien).

Im Übrigen haben Dänemark, die Niederlande, Österreich und die Schweiz für die gleiche Zusammenstellung aus fünf LB MMV festgelegt. Schließlich haben für die LB Pankreas, Ösophagus, Leber und Kolorektale Eingriffe insgesamt sieben Länder MMV definiert, während für die LB Bariatrische Eingriffe, Mammakarzinom und Geburtshilfe nur sechs bzw. fünf und drei Länder MMV festlegen. Für den LB Cholezystektomie gilt eine MMV nur in Italien. 


\section{Anwendungsebene der MMV}

Alle Länder haben MMV auf Krankenhausebene für ihre jeweiligen LB eingeführt. Für ausgewählte LB bestehen in Deutschland (DKG-MMV für Pankreas, Ösophagus, Kolorektale Eingriffe; DKG- und DMP-MMV für Mammakarzinom), Großbritannien (Pankreas, Ösophagus, Leber, Bariatrische Eingriffe) und Dänemark (s. Großbritannien zzgl. Kolorektale Eingriffe) MMV auf Operateurebene. Darüber hinaus gelten ab 2019 erstmalig auch in der Schweiz im Kanton Zürich Mindestfallzahlen pro Operateur.

\section{Regulierungsabsicht}

In einer ersten Recherche wurde festgestellt, dass die untersuchten Länder MMV für vier Absichten verwenden, die aufsteigend gemäß ihrer regulativen Härte und damit Auswirkung auf den Zentralisierungsgrad aufgeführt werden:

- Zertifizierung (z.B. Deutschland): Diese werden von Krankenhäusern freiwillig erworben, um eine hohe Qualifizierung für die Behandlung einer bestimmten Krankheit oder eines Organ(-systems) nachweisen zu können. Ein zentrales Kriterium ist hierfür das Erreichen einer Mindestzahl an Eingriffen bzw. Behandlungen. Der so entstehende Qualitätswettbewerb hat bei ausreichender Qualitätstransparenz zur Folge, dass Patienten und Einweiser nur solche Zentren auswählen, die zertifiziert sind, was langfristig eine Zentralisierung der Leistungen zur Folge hat.

- Vergütung (z.B. Deutschland): Ein erbrachter Eingriff bzw. eine durchgeführte Behandlung wird nur dann vergütet, wenn die MMV für diesen Eingriff bzw. diese Behandlung eingehalten wurde. Durch eine fehlende Vergütung sind also solche Krankenhäuser, die die MMV nicht erreichen können, dazu angehalten, entsprechende Kapazitäten abzubauen und Patienten nicht weiter zu behandeln. Mittel- bis langfristig werden diese Patienten in den Zentren behandelt, die der MMV entsprechen.

- Leistungsvergabe (z.B. Frankreich, Niederlande): MMV werden genutzt, um Krankenhäuser zu identifizieren, die als Partner für Selektivverträge bzw. als Leistungserbringer für komplexe Eingriffe geeignet sind. Hierdurch entsteht mittelfristig eine schrittweise Zentralisierung dieser Leistungen.

- Kapazitätsplanung (z.B. Italien, Niederlande, Österreich, Schweiz): Indem nur solche Krankenhäuser Kapazitäten zugesprochen bekommen (z.B. Betten für eine Fachabteilung), die eine bestimmte MMV für einen LB erfüllen (Strukturkriterium für Kapazitätsvergabe), soll eine kurzfristige, wirkungsvolle Zentralisierung von Krankenhauskapazitäten herbeigeführt werden. 


\section{Höhe der Schwellenwerte}

Die Höhe der Schwellenwerte je LB unterscheidet sich stark zwischen den untersuchten Ländern. In Dänemark und England gelten generell relativ hohe Schwellenwerte, was auf stärkere Zentralisierungsbemühungen dieser beiden Länder hindeutet. In Österreich sind die Schwellenwerte insgesamt am niedrigsten angesetzt. Auffällig ist, dass die beiden durch den G-BA gesetzten Schwellenwerte für Pankreas und Ösophagus genauso hoch wie die Schwellenwerte in Österreich und damit im Vergleich ebenfalls am niedrigsten sind. Die in Deutschland gültigen Zertifizierungsvorgaben fordern demgegenüber vergleichbar hohe Schwellenwerte wie beispielsweise in Frankreich und liegen damit im Mittelfeld.

\section{Grad der Durchsetzung und Ausnahmeregelungen}

Während in Deutschland eine Reihe an Ausnahme- und Übergangsregelungen gelten, bestehen in den Vergleichsländern generell weniger Möglichkeiten, MMV zu umgehen. In Frankreich besteht zum Beispiel lediglich die Ausnahme, dass bei lückenhafter Versorgungsdichte die jeweilige MMV ausgesetzt werden kann, was jedoch anhand des an der Bevölkerung abgeleiteten Bedarfs nachgewiesen werden muss. In der Schweiz wird zur Prognose der Fallzahl und Einhaltung der MMV der 2-Jahresdurchschnitt des jeweiligen LBs herangezogen. Sollte dieser Durchschnitt erstmalig unter dem Schwellenwert liegen, darf das jeweilige Krankenhaus die Leistung unter Vorbehalt ein weiteres Jahr erbringen. Diese Erlaubnis wird im Folgejahr nicht noch einmal vergeben, sollte die MMV ein weiteres Mal nicht eingehalten worden sein. Da in den Niederlanden MMV von mehreren, teilweise privaten Organisationen definiert werden, konnte kein klares Muster an Ausnahmen festgestellt werden. Generell haben aber Krankenversicherungen den Anreiz, relativ hohe MMV mit wenigen Ausnahmen zu definieren, um im (Qualitäts-) Wettbewerb um Versicherte glaubhaft höhere Ergebnisqualität versprechen zu können.

\subsubsection{Modellierung europäischer Mindestmengenvorgaben}

Für die vorliegende Analyse wurde für die LB Pankreas und Ösophagus die G-BA-Definition verwendet. Für die LB Leber und Kolorektale Eingriffe wurde die DKG-Definition und für Mammakarzinom die entsprechende DMP- bzw. DKGDefinition genutzt. Für den LB Cholezystektomie wurde die WIdO-Definition angewendet und für den LB Geburtshilfe wurde eine eigene, allgemeine Definition verwendet (s. Tab. 1). Für die jeweiligen LB wurden dann die entsprechenden Schwellenwerte der Vergleichsländer (s. Achsenbeschriftung Abb. 2) genutzt, um die Anzahl der Krankenhäuser in Deutschland, die der jeweiligen MMV entsprechen, und die durchschnittliche Anzahl der Eingriffe je Krankenhaus zu berechnen. 


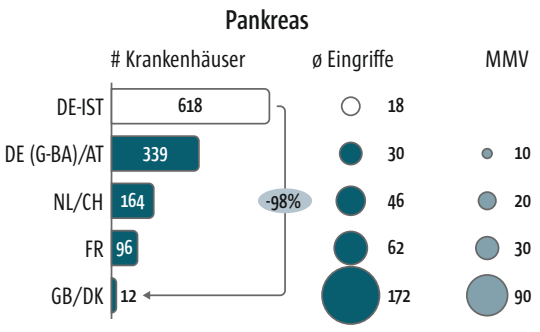

Leber

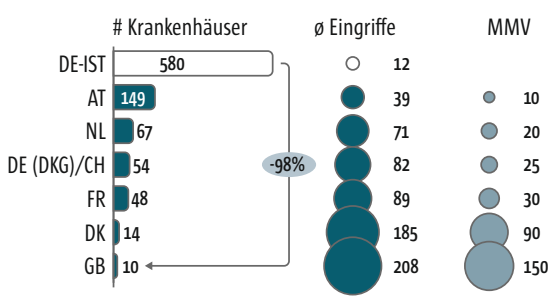

Bariatrische Eingriffe

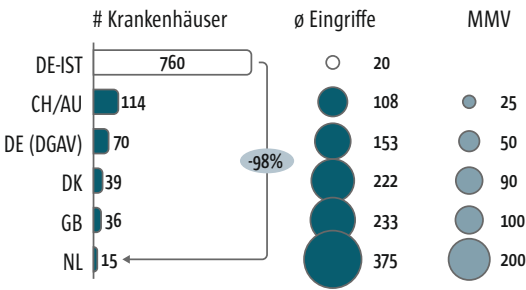

Mammakarzinom

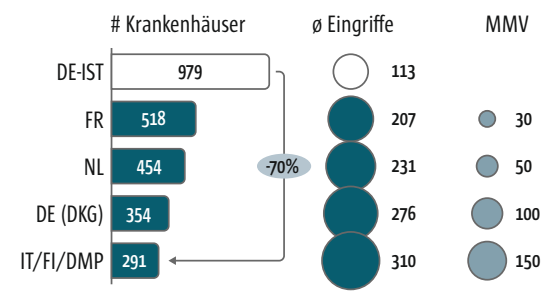

Ösophagus

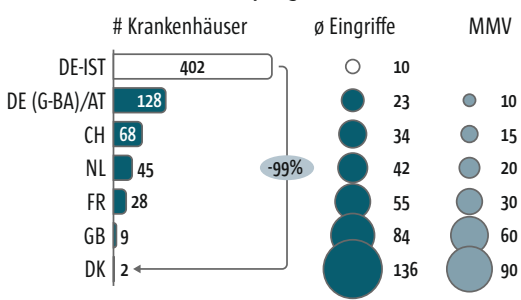

Kolorektale Eingriffe

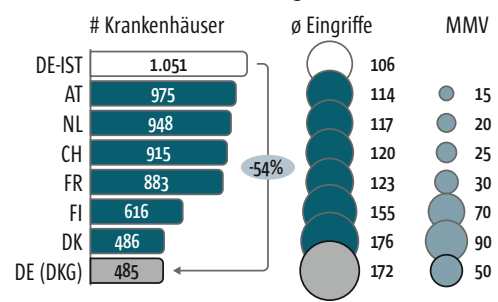

Cholezystektomie

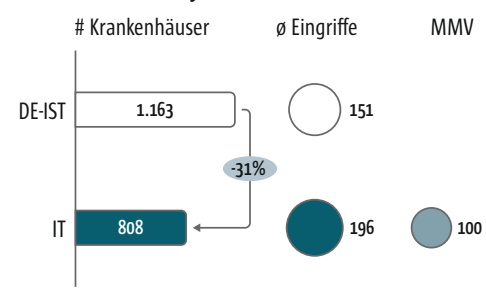

Geburtshilfe

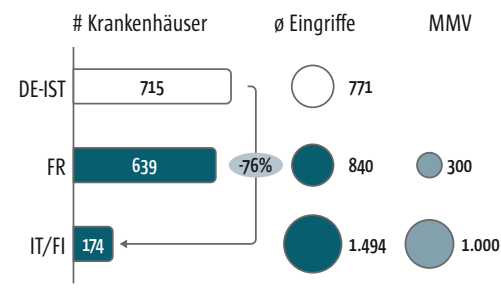

Abb. 2 Anzahl an Krankenhäusern, die unterschiedlichen deutschen MMV auf Krankenhausebene entsprechen. Hinweise: (1.) Länderkürzel gem. internationaler Klassifizierung (2.) Die durchschnittliche Anzahl der Eingriffe für „mit MMV“ bezieht sich nur auf die Eingriffe, die von den Krankenhäusern tatsächlich erbracht wurden. Eingriffe, die von Krankenhäusern erbracht wurden, die der MMV nicht entsprechen, wurden nicht auf die verbleibenden Krankenhäuser verteilt. (3.) Für die MMV der DKG handelt es sich lediglich um eine Abbildung nach Teilkriterien (OPS-Codes). Für eine vollständige Darstellung sind ICD- und OPS-Codes auf Patientenebene nötig.

Insgesamt sind die Schwellenwerte je LB von anderen Ländern wesentlich höher angesetzt als in Deutschland und entsprechend werden die verschiedenen MMV nur von einem Bruchteil der Krankenhäuser in Deutschland eingehalten

urheberrechtlich geschützt, 
(s. Abb. 2). Besonders signifikant ist der Effekt für Pankreas, Ösophagus, Leber und Bariatrische Eingriffe, am schwächsten ist der Effekt einer MMV für Cholezystektomie. Wenig überraschend gründet dieser Umstand v.a. in der Höhe des Schwellenwerts für die jeweiligen LB und die Komplexität des LBs an sich. Neben den in der Methodik beschriebenen Limitationen sollte zusätzlich beachtet werden, dass in der Analyse jeweils die in Abbildung 2 angegebenen Definitionen je LB angewendet wurden und in der limitierten Recherchedauer nicht abschließend geklärt werden konnte, zu welchem Grad die Definitionen der LB der Vergleichsländer sich von den verwendeten Definitionen unterscheiden (generell stellt die Definition der LB inkl. Anzahl Eingriffsarten die siebte Ebene von MMV dar). Entsprechend wurde die Definition je LB gewählt, die am allgemeinsten formuliert ist (4-stellige und/oder viele 5-stellige OPSCodes) um eine generelle Vergleichbarkeit herzustellen und die verschiedenen Schwellenwerte eines LBs für die Analyse verwenden zu können. Darüber hinaus sind die Kodiersysteme im Allgemeinen in Europa äußerst heterogen, weshalb eine Vergleichbarkeit von LBs z.B. anhand von Prozedurencodes derzeit nicht gegeben ist (Geissler et al. 2015). Einige internationale Projekte, wie z.B. das ICD-9 CM Matching der OECD oder das Hospital Data Project (Hospital Data Project 2008; Lorenzoni u. Marino 2017), haben einen Abgleich verschiedener europäischer Definitionen von Diagnosen und Prozeduren bzw. LB vorgelegt. Diese Matching-Ansätze können jedoch nicht direkt auf die vorliegende Analyse übertragen werden, da sich die Kodiersysteme in der Zwischenzeit erneuert haben, nicht alle hier betrachteten LB untersucht wurden und/oder andere Klassifizierungssysteme für das Matching genutzt wurden.

Ungeachtet dessen bietet die Analyse interessante Erkenntnise, wovon die folgenden drei näher beleuchtet werden sollen:

- Die DKG-MMV für Kolorektale Eingriffe hat den signifikantesten Effekt, obwohl der aggregierte Schwellenwert nicht der höchste ist. Dies liegt daran, dass die DKG-MMV Schwellenwerte für zwei Eingriffsarten umfasst (30 Kolonresektionen und 20 Rektumresektionen). Da eine Aufteilung der Mengen zwischen diesen beiden Eingriffsarten für die Vergleichsländer nicht angegeben bzw. nicht vorhanden ist, wurden die Schwellenwerte der Vergleichsländer auf die Summe aller Kolon- und Rektumresektionen angewendet. Dies unterstreicht die Erkenntnis des letzten Kapitels, dass neben dem (aggregierten) Schwellenwert auch die Anzahl der Eingriffsarten großen Einfluss auf den (potenziellen) Zentralisierungsgrad hat.

- Die Anzahl der Krankenhäuser, die mindestens 1 Eingriff des LBs Bariatrische Eingriffe durchführen, ist gemessen an der Anzahl der Krankenhäuser, die schon eine MMV von 25 Eingriffen nicht mehr erfüllen, relativ hoch. Dies ist u.a. auf die breite DGAV-Definition dieses LBs zurückzuführen (s. Tab. 1), unter die auch allgemeinere Eingriffe fallen (z.B. 5-467.5: Andere Rekonstruktion des Darmes - Revision einer Anastomose). 
- Lediglich etwas mehr als 170 Krankenhäuser in Deutschland würden einer Geburtshilfe-MMV mit einem Schwellenwert von 1.ooo Lebendgeburten entsprechen (durchschnittlich weniger als 3 Geburten pro Tag). Darüber hinaus führen ca. 75 Krankenhäuser weniger als durchschnittlich 1 Geburt pro Tag durch und würden dementsprechend einer MMV mit einem Schwellenwert von 300 Lebendgeburten nicht gerecht werden.

\subsection{Zusammenfassung}

Aus der vorgenommenen quantitativen Analyse lässt sich ableiten, dass (1.) trotz bestehender MMV in Deutschland weiterhin eine starke Streuung der Leistungserbringung vorhanden ist, gerade auch für komplexe LB wie Pankreas oder Ösophagus, dass (2.) nur ein Bruchteil der Krankenhäuser im Untersuchungsjahr die MMV des G-BA, verschiedener Fachgesellschaften und europäischer Vergleichsländer erfüllt hätte, (3.) eine strikte Durchsetzung dieser MMV entsprechend signifikante Auswirkungen auf den stationären Krankenhaussektor hätte und dass (4.) der Effekt der strikten Durchsetzung einer MMV von der Definition des LBs, der Anzahl der Eingriffsarten mit Schwellenwerten und der Höhe des (jeweiligen) Schwellenwerts abhängt. Zu (2.) muss limitierend hinzugefügt werden, dass eine Umleitung der Patienten von Krankenhäusern unter den jeweiligen Schwellenwerten hin zu Krankenhäusern, die die jeweilige $M M V$ erfüllen, nicht berücksichtigt wurde.

Im Übrigen ergeben sich durch die qualitative Analyse der verschiedenen MMV folgende sieben Erkenntnisse:

1. Definition der LB: Abgesehen von den Klarnamen der Leistungsbereiche besteht keine einheitliche Definition der LB. In Deutschland basieren die Definitionen auf ICD-Codes, OPS-Codes oder einer Kombination dieser Codes.

2. Art der erlassenden Behörde: MMV werden von Bundesministerien bzw. deren Stellvertretern auf bundesweiter und/oder regionaler Ebene, Fachgesellschaften und Krankenversicherungen erlassen. Die Art der erlassenden Behörde hat Einfluss auf die Definition der LB, die Regulierungsabsicht, die Höhe der Schwellenwerte und auf den Umfang und die Art der Ausnahmeregelungen.

3. Anzahl der LB: Jedes der untersuchten Länder nutzt MMV, jedoch variiert die Anzahl und die Zusammenstellung der LB zwischen den Ländern.

4. Anwendungsebene der MMV: MMV werden sowohl auf Krankenhausebene als auch auf Operateursebene eingesetzt.

5. Regulierungsabsicht: MMV werden festgelegt, um Zertifizierungen zu vergeben, Vergütung von Leistungen zu regeln, die Leistungsvergabe z.B. durch Selektivverträge zu steuern und Kapazitäten zu planen. Während Zertifizierungen durch einen verstärkten Qualitätswettbewerb die Krankenhauslandschaft eher indirekt und langfristig beeinflussen, sind 
die übrigen Regulierungen mit zunehmenden Grad direkte Eingriffe in die Versorgungsstruktur.

6. Höhe der Schwellenwerte: Die Höhe der Schwellenwerte je LB variiert beträchtlich zwischen den untersuchten Ländern und Organisationen. Generell steht die Höhe des Schwellenwertes für den beabsichtigten Zentralisierungsgrad bzw. repräsentiert die Menge, um ein Zentrum der „Spitzenmedizin“ auszuzeichnen.

7. Ausnahmeregelungen: Während der G-BA eine Reihe von Ausnahmeregelungen vorsieht, wurde Vergleichbares für andere deutsche und europäische MMV nicht im gleichen Umfang gefunden. Die bisher mäßige Zentralisierung komplexer Leistungsbereiche könnte auf diese Regelungen zurückzuführen sein.

Vor dem Hintergrund dieser Ergebnisse sollte unter Berücksichtigung der jeweiligen Regulierungsabsichten eine einheitlichere Definition der LBs geprüft werden, um die Vergleichbarkeit der MMV zu erhöhen. Außerdem sollte kontinuierlich geprüft werden, ob die Höhe der Schwellenwerte der Regulierungsabsicht weiterhin entspricht (vgl. Begleitforschung der Einführung von Mindestmengen in Deutschland). Schließlich sollte überprüft werden, (1) ob MMV auf Operateursebene die jeweilige Regulierungsabsicht unterstützen können, (2) ob und welche Ausnahmeregelungen der Regulierungsabsicht im Wege stehen könnten und (3) ob nicht noch weitere Leistungsbereiche für MMV in Deutschland infrage kämen.

Gemäß der oben aufgeführten und in der Methodik beschriebenen Limitationen, werden folgende Weiterentwicklungen der Modellierung in einem umfassenden Modell empfohlen, um die Erkenntnisse dieses Beitrags zu festigen und zu erweitern sowie die Wirksamkeit von MMV als gesundheitspolitische Maßnahme weiter zu prüfen:

- Umverteilung der Patienten, die in von der Versorgung ausgeschlossenen Krankenhäusern behandelt wurden

- Auswirkungen des Ausschlusses von Krankenhäusern gemäß MMV auf die Patientenreisezeit

- Berechnung des positiven Effekts auf die Ergebnisqualität je Krankenhaus und auf Systemebene durch MMV

- Berechnung der Zentralisierung von Kapazitäten und damit Ressourceneinsparung durch MMV

Eine Modellierung, die alle obigen Punkte einschließt, könnte beispielsweise mit den Daten der DRG-Statistik auf Patientenebene und insbesondere mit Standortbezug durchgeführt werden. Diese Daten sind der Forschung jedoch zum jetzigen Zeitpunkt nicht vollumfänglich zugänglich. Die verwendeten Daten der externen stationären Qualitätssicherung sind für Analysen dieser Art nicht vorgesehen und besitzen ein zu hohes Aggregationsniveau. 


\section{Take home messages}

- quantitative Analyse: Es besteht eine starke Streuung der Leistungserbringung in Deutschland, die je nach MMV unterschiedlich stark zentralisiert würde.

- qualitative Analyse: MMV in Deutschland und den acht untersuchten europäischen Ländern unterscheiden sich auf sieben Ebenen, die alle direkten oder indirekten Einfluss auf den potenziellen Zentralisierungseffekt der MMV haben.

- Weiterentwicklung: Es sollte ein Modell vorgelegt werden, das die Auswirkungen von MMV in Deutschland realistisch anhand der Variablen erzielte Ergebnisqualität, Patientenreisezeit und Krankenhauskapazität simuliert. Optimale Datengrundlage hierfür sind die Daten der DRG-Statistik auf Patientenebene mit Standortbezug.

\section{Literatur}

Allum WH, Blazeby JM, Griffin SM, Cunningham D, Jankowski JA, Wong R (2011) Guidelines for the management of oesophageal and gastric cancer. URL: http://www.augis.org/wp-content/uploads/2014/05/Gut-2011Allum-gut-2010-228254.pdf (abgerufen am 11. Juni 2018)

Ärztekammer Berlin (2016) Logbuch Facharztweiterbildung Frauenheilkunde und Geburtshilfe. URL: https:// www.aerztekammer-berlin.de/10arzt/15_Weiterbildung/11Logbuecher/Facharzt-Weiterbildung/Uebersicht_LB_Frauenheilkunde_und_Geburtshilfe/Logbuch_FA_Frauenheilkunde_Geburtshilfe_1_-7_NT.pdf (abgerufen am 06. Juni 2018)

AUGIS - Association of Upper Gastrointestinal Surgeons of Great Britain and Ireland (2010) Guidance on Minimum Surgeon Volumes. URL: http://www.augis.org/wp-content/uploads/2014/05/AUGIS_recommendations_on_Minimum_Volumes.pdf (abgerufen am 11. Juni 2018)

Blum K, Offermanns M (2006) Auswirkungen der DMP Brustkrebs auf die Krankenhäuser. Studie des Deutschen Krankenhausinstituts (DKI) im Auftrag der Deutschen Krankenhausgesellschaft (DKG). URL: https://www. dki.de/sites/default/files/publikationen/bericht_dmp.pdf (abgerufen am 11. Juni 2018)

Chowdhury M, Dagash H, Pierro A (2007) A systematic review of the impact of volume of surgery and specialization on patient outcome. Br I Surg 94(2), 145-161.

de Cruppé W, Geraedts M (2016) Wie konstant halten Krankenhäuser die Mindestmengenvorgaben ein? Eine retrospektive, längsschnittliche Datenanalyse der Jahre 2006, 2008 und 2010. Zentralbl Chir 141(04), 425-432.

de Cruppé W, Geraedts M (2018) Mindestmengen unterschreiten, Ausnahmetatbestände und ihre Konsequenzen ab 2018. Komplexe Eingriffe am Ösophagus und Pankreas in deutschen Krankenhäusern im Zeitverlauf von 2006 bis 2014. Zentralbl Chir 143(3), 250-258.

de Cruppé W, Malik M, Geraedts M (2014) Achieving minimum caseload requirements: an analysis of hospital quality control reports from 2004-2010. Dtsch Arztebl Int 111(33-34), 550-555.

Deutsche Gesellschaft für Allgemein- und Viszeralchirurgie e.V. (2018) Zertifizierungen der DGAV. URL: http:// dgav.de/savc/zertifizierungen.html (abgerufen am 20. Juni 2018)

Geissler A, Lee S, Quentin W (2017) Der Einsatz von Qualitätsinformationen für Krankenhausplanung und Leistungseinkauf in Frankreich, Italien, Niederlande, Österreich und der Schweiz. In: Klauber, Geraedts, Friedrich, Wasem, eds. (2017) Wissenschaftliches Institut der AOK (WId0), Krankenhaus-Report 2017, Schwerpunkt: Zukunft gestalten. Schattauer-Verlag, Stuttgart.

Geissler A, Quentin W, Busse R (2015) Heterogeneity of European DRG systems and potentials for a common EuroDRG system: Comment on "Cholecystectomy and Diagnosis-Related Groups (DRGs): patient classification and hospital reimbursement in 11 European countries." Int J Health Policy Manag 4(5), 319-320. 
Gemeinsamer Bundesausschuss (2018) Regelungen des Gemeinsamen Bundesausschusses gemäß $§ 136 \mathrm{~b}$ Absatz 1 Satz 1 Nummer 2 SGB V für nach $\ 108$ SGB V zugelassene Krankenhäuser. In: Mindestmengenregelungen. URL: https://www.g-ba.de/downloads/62-492-1601/Mm-R_2018-05-17_iK-2018-06-08.pdf (abgerufen am 12. Mai 2018)

Geraedts M, De Cruppe W, Blum K, Ohmann C (2010) Distanzen zu Krankenhäusern mit Mindestmengen-relevanten Eingriffen 2004 bis 2006. Das Gesundheitswesen 72(05), 271-278.

Hillienhof A (2018) Mindestmengen: Wahrscheinliche Zusammenhänge reichen aus. Dtsch Arztebl 115(24), 1149 .

Hospital Data Project (2008) Phase 2 - Final Report: The equal need for metadata and data. URL: http://ec.europa. eu/health/ph_projects/2004/action1/docs/action1_2004_frep_32_en.pdf (abgerufen am 18. August 2018)

Italia (2012) Gli Standard per La Valutazione dei Punti Nascita. URL: http://www.salute.gov.it/imgs/C_17_pagine Aree_4483_listaFile_itemName_2_file.pdf (abgerufen am 12. Juni 2018)

Italia (2015) Decreto 2 aprile 2015, n. 70, Regolamento recante defi nizione degli standard qualitativi, strutturali, tecnologici e quantitativi relativi all'assistenza ospedaliera. Gazzetta Ufficiale. URL: http://www. gazzettaufficiale.it/eli/id/2015/06/04/15G00084/sg (abgerufen am 05. Juni 2018)

Jüni P, Hossmann S, Rat J, Limacher A, Rutjes AWS (2014) Interkantonale Vereinbarung zur hochspezialisierten Medizin (IVHSM): Seltenheit als Kriterium für die Konzentration der hochspezialisierten Medizin. Institut für Sozial- und Präventivmedizin, Universität Bern. URL: https://www.gdk-cds.ch/fileadmin/docs/public/ gdk/themen/hsm/organe/hsm_finalreport_executivesummary_version_pi_20140508_dt.pdf (abgerufen am 14. Juni 2018)

KCE - Federaal Kenniscentrum voor de Gozondheidszork (2017) Required Hospital Capacity in 2025 and Criteria for Rationalisation of Complex Cancer Surgery, Radiotherapy and Maternity Services. In: KCE Report 289. URL: https://kce.fgov.be/sites/default/files/atoms/files/Download\%20het\%20rapport\%20in\%20het\%20 Engels\%20\%28550\%20p.\%29.pdf (abgerufen am 01. Juli 2018)

Loos S, Albrecht M, Schiffhorst G, Ochmann R, Möllenkamp N (2016) Faktencheck Krankenhausstruktur. Spezialisierung und Zentrenbildung. In: IGES/Bertelsmann Stiftung. URL: https://www.bertelsmann-stiftung.de/ fileadmin/files/Projekte/Faktencheck_Gesundheit/FC_Krankenhausstruktur_Studie_final.pdf (abgerufen am 12. Juni 2018)

Lorenzoni L, Marino A (2017) Understanding variations in hospital length of stay and cost: Results of a pilot project. OECD Health Working Papers, No. 94

Luft HS, Bunker JP, Enthoven AC (1979) Should operations be regionalized? The empirical relation between surgical volume and mortality. N Engl J Med 301(25), 1364-1369.

Luft HS, Hunt SS, Maerki SC (1987) The Volume-Outcome-Relationship: Practice-Makes-Perfect or SelectiveReferral Patterns? Health Serv Res 22(2), 157-182.

Mansky T, Nimptsch U, Cools A, Hellerhoff F (2016) G-IQI - German Inpatient Quality Indicators : Version 5.0 Band 2: Definitionshandbuch für das Datenjahr 2016. doi: 10.14279/depositonce-5424

Markar SR, Karthikesalingam A, Thrumurthy S, Low DE (2012) Volume-Outcome Relationship in Surgery for Esophageal malignancy: Systematic Review and Meta-analysis 2000-2011. I Gastrointest Surg 16(5), 1055-1063.

National Health Service (NHS) (2018) Highly specialized services 2017. URL: https://www.england.nhs.uk/commissioning/spec-services/highly-spec-services/ (abgerufen am 12. Juni 2018)

Nederlandse, Inspectie voor de Gezondheidszork, Ministerie van Volksgezondheid, Welzijn en Sport (2017) Het resultaat telt ziekenhuizen 2015. URL: https://www.rijksoverheid.nl/documenten/rapporten/2017/02/22/ het-resultaat-telt-ziekenhuizen-2015 (abgerufen am 12. Juni 2018)

Nederlandse Vereniging voor Heelkunde (2017) Normering Chirurgische Behandelingen 7.0. URL: https://heelkunde.nl/sites/heelkunde.nl/files/Normen\%207.0\%20definitief_1.pdf (abgerufen am 12. Juni 2018)

Nimptsch U, Mansky T (2018) Volume-Outcome-Zusammenhänge in Deutschland. In: Dormann F, Klauber I, Kuhlen R (Hrsg.) Qualitätsmonitor 2018, 55-68. Medizinisch Wissenschaftliche Vertragsgesellschaft Berlin.

Nobilio L, Fortuna D, Vizioli M, Berti E, Guastaroba P, Taroni F, Grilli R (2004) Impact of regionalization of cardiac surgery in Emilia-Romagna, Italy. I Epidemiol Community Health 58, 97-102.

Onkozert (2018) Zertifizierung durch Onkozert. URL: http://www.onkozert.de/index.htm (abgerufen am 12. Mai 2018)

urheberrechtlich geschützt, 
ÖSG - Der Österreichische Strukturplan Gesundheit 2017 (2017). Leistungsmatrix stationär 2019, Stand 29.06.2018. URL: https://www.bmgf.gv.at/home/Gesundheit/Gesundheitssystem_Qualitaetssicherung/Planung_und spezielle_Versorgungsbereiche/Der_Oesterreichische_Strukturplan_Gesundheit_ndash_OeSG_2017 (abgerufen am 12. Juni 2018)

Peschke D, Nimptsch U, Mansky T (2014) Achieving minimum caseload requirements - an analysis of hospital discharge data from 2005-2011. Dtsch Arztebl Int 111(33-34), 556-563.

République Française, Ministère de la Santé et des Sports (2011) Guide méthodologique pour l'élaboration du SROS-PRS. URL: http://solidarites-sante.gouv.fr/IMG/pdf/Guide_SROS.pdf (abgerufen am 12. Juni 2018)

Russo MJ, Iribarne A, Easterwood R (2010) Post-heart transplant survival is inferior at low-volume centers across all risk strata. Circulation 122(11), 85-91.

Sachverständigenrat zur Begutachtung der Entwicklung im Gesundheitswesen (2018) Bedarfsgerechte Steuerung der Gesundheitsversorgung. URL: https://www.svr-gesundheit.de/fileadmin/user_upload/Gutachten/2018/SVR-Gutachten_2018_WEBSEITE.pdf (abgerufen am 25. Juli 2018)

SPLG - Spitalplanungs-Leistungsgruppen Kanton Zürich (2018) URL: https://www.gdk-cds.ch/index.php?id=822 (abgerufen am 12. Juni 2018)

Suomi, Valtioneuvosto (2017) Valtioneuvoston asetus erikoissairaanhoidon työnjaosta ja eräiden tehtävien keskittämisestä, 24.8.2017/582 sowie Valtioneuvosto. Valtioneuvoston asetus kiirellisen hoidon perusteista ja päivystyksen erikoisalskohtaisista edellytyksistä. URL: https://www.finlex.fi/fi/laki/alkup/2017/20170582 (abgerufen am 12. Juni 2018)

Wissenschaftliches Institut der AOK (2017) Indikatorenhandbuch für Leistungsbereiche mit Berichterstattung im AOK-Krankenhausnavigator, Verfahrensjahr 2017. URL: http://www.qualitaetssicherung-mit-routinedaten.de/imperia/md/qsr/methoden/wido_qsr_indikatorenhandbuch_mit_bericht_2017.pdf (abgerufen am 18. Juni 2018)

Zorguerzekeraars Nederland (2017) Minimum kwaliteitsnormen ziekenhuizen. URL: https://www.minimumkwaliteitsnormen.nl/Normen.htm (abgerufen am 26. Juni 2018)

\section{Danksagungen}

Wir bedanken uns bei unseren Experten Florian Bachner (Gesundheitsökonomie und -systemanalyse, Gesundheit Österreich $\mathrm{GmbH}$ ), Florien Kruse (Scientific Center for Quality of Healthcare, Netherlands), Bruno Lucet (Direction de l'Amélioration de la Qualité \& de la Sécurité des Soins), Marianna Mauro (Associate Professor of Business Economics University of Catanzaro) und Dominik Moser (Technische Universität Berlin) für die geführten Interviews. 


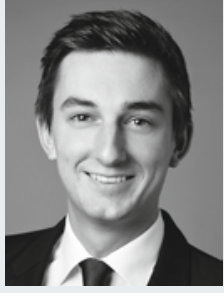

Justus Vogel, M.Sc.

Bachelor- und Masterstudium von 2010 bis 2015 an der WHU - Otto Beisheim School of Management. Im Anschluss mehrjährige Beschäftigung bei der Boston Consulting Group mit Fokus auf Beratung von Unternehmen des Gesundheitssektors. Seit Februar 2018 Wissenschaftlicher Mitarbeiter im Projekt Qualitätstransparenz und deren Auswirkung auf die Qualität stationärer Versorgung des Fachgebiets Management im Gesundheitswesen der Technischen Universität (TU) Berlin.

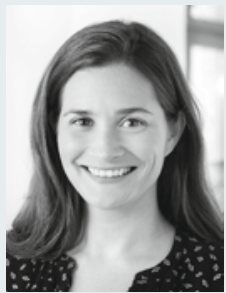

\section{Katherine Polin, MPH}

Nach Beendigung ihres Studiums der Geisteswissenschaften (Schwerpunkt: vergleichende Literatur) an der Yale University, arbeitete sie für Organisationen im Bereich Gesundheitswesen, Menschenrecht und Systemverwaltung. Als Council of Women World Leaders Fellow 2012 wertete sie für das Bundesministerium für Familien, Senioren, Frauen und Jugend Primärversorgungsinitiativen bzgl. der Gewalt gegen Frauen aus. 2013 schloss sie ihre Masterarbeit im Bereich Gesundheitswesen an der Columbia University ab. 2014 und 2015 Arbeit als Transatlantic Fellow der Robert Bosch Stiftung in Berlin. Danach mehrjährige Arbeit für die Beratungsfirma SEEK Development und seit Februar 2018 Wissenschaftliche Mitarbeiterin im Projekt Qualitätstransparenz und deren Auswirkung auf die Qualität stationärer Versorgung des Fachgebiets Management im Gesundheitswesen der TU Berlin.

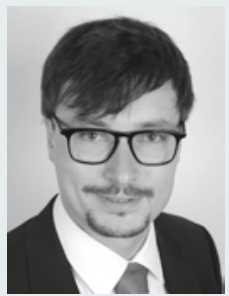

\section{Dr. Christoph Pross}

Nach dem VWL-Studium am Davidson College, USA, und der London School of Economics arbeitet er seit 2011 für die Boston Consulting Group in der Beratung von Unternehmen des Gesundheitssektors. Von 2014 bis 2017 promovierte er an der TU Berlin zum Thema Qualitätstransparenz und deren Auswirkung auf die Qualität stationärer Versorgung und co-leitet aktuell das gleichnamige Forschungsprojekt an der TU Berlin.

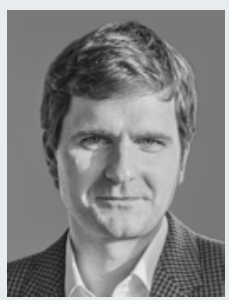

\section{PD Dr. Alexander Geissler}

Wissenschaftlicher Mitarbeiter am Fachgebiet Management im Gesundheitswesen der TU Berlin. Dort leitet er den Arbeitsbereich stationäre Versorgung. Er ist promovierter Gesundheitsökonom und habilitiert für die Fächer Gesundheitssystemforschung und Gesundheitsökonomie an der TU Berlin. Seine Forschung beschäftigt sich mit Anreizund Vergütungssystemen von Krankenhäusern und deren Auswirkungen auf die Qualität und Wirtschaftlichkeit der Versorgung.

urheberrechtlich geschützt, 